\title{
Diretrizes do distúrbio do metabolismo mineral e ósseo na doença renal crônica da criança
}

Brazilian Guidelines for bone and mineral disorders in CKD children

\section{Avaliação do metabolismo mineral}

1.1 Os níveis séricos de cálcio $(\mathrm{Ca})$, fósforo $(\mathrm{P})$, fosfatase alcalina (FA), paratormônio-intacto (PTH), $\mathrm{pH}$ e bicarbonato sérico $\left(\mathrm{HCO}_{3}\right)$ ou reserva alcalina $\left(\mathrm{CO}_{2}\right.$ total $)$ devem ser determinados em todas as crianças e adolescentes nos estágios II a $\mathrm{V}$ da doença renal crônica (DRC). A frequência dessas determinações deve ser baseada na presença e na magnitude das alterações e na velocidade de progressão da DRC (Tabela 1) (Opinião).

1.2 Estas medidas devem ser mais frequentes se o paciente está recebendo tratamento para as alterações dos níveis séricos de $\mathrm{Ca}, \mathrm{P}, \mathrm{PTH}$ ou 25-hidroxivitamina $\mathrm{D}$, está em uso de hormônio de crescimento ou foi submetido a transplante renal (Opinião).
1.3 Os níveis-alvo de PTH nos diversos estágios da DRC estão apresentados na Tabela 2.

Em crianças e adolescentes com DRC estágios II a V, os níveis de 25 -vit D (calcidiol) devem ser medidos e a frequência dessas medidas deve ser determinada pelos valores basais e pelas intervenções terapêuticas. A deficiência de 25-hidroxivitamina $\mathrm{D}$ deve ser corrigida usando as recomendações de tratamento para a população geral (diretriz 5) (Opinião).

\section{RACIONAL}

As alterações do metabolismo mineral e da estrutura óssea são universais na doença renal crônica (DRC) na infância e resulta em muitas complicações. $\mathrm{Na}$ criança, a osteodistrofia renal (OR) causa, como nos adultos, muitas complicações

\begin{tabular}{|c|c|c|c|c|}
\hline Tabela 1 & \multicolumn{4}{|c|}{$\begin{array}{l}\text { FREQUÊNCIA DAS DETERMINAÇÕES DE CA, } \mathrm{P}, \mathrm{FA}, \mathrm{PTH}, \mathrm{PH} \text { E } \mathrm{HCO}_{3} \text { OU } \mathrm{CO}_{2} \\
\text { TOTAL DE ACORDO COM O ESTÁGIO DA DRC }\end{array}$} \\
\hline $\begin{array}{l}\text { Estágio da } \\
\text { DRC }\end{array}$ & $\begin{array}{l}\mathrm{TFG} *(\mathrm{~mL} / \\
\mathrm{min} / 1,73 \mathrm{~m} 2)\end{array}$ & $\mathrm{Ca}, \mathrm{P}, \mathrm{pH}, \mathrm{HCO}_{3}$ ou $\mathrm{CO}_{2}$ total & FA e PTH & Calcidiol \\
\hline II & $60-89$ & Semestral a anual & Semestral a anual & Basal \\
\hline III & $30-59$ & Quadrimestral & Quadrimestral & Basal \\
\hline IV & $15-29$ & Trimestral & Trimestral & Basal \\
\hline V & < 15 ou diálise & Mensal & Trimestral & Basal \\
\hline
\end{tabular}

*TFG = taxa de filtração glomerular - Cálculo por fórmula de Schwartz (Opinião).

Tabela 2 NíveIS DE PTH dE ACORDO COM O ESTÁGIO DA DRC

Nível sérico de PTH

35-70 (Opinião)

35-70 (Opinião)

70-110 (Opinião)

15-29

$<15$ ou diálise

200-300 (Evidência) 
como fraturas, dor óssea e necrose vascular, mas o retardo de crescimento e as deformidades ósseas são características das crianças. ${ }^{1} \mathrm{~A}$ associação entre o distúrbio do metabolismo mineral, fraturas, doença cardiovascular (DCV) e mortalidade observada em adulto e crianças ${ }^{2}$ levou à reclassificação das complicações bioquímicas, esqueléticas e vasculares associadas com a doença renal progressiva, sendo denominadas de distúrbio mineral e ósseo da doença renal crônica (DRC-DMO). A retenção de P ocorre em estágios precoces da DRC da criança (estágio II) e tem um papel importante para o desenvolvimento do hiperparatireoidismo secundário. Embora a redução dos níveis de 1,25-vit D (calcitriol) tenha sido considerada como o evento inicial para a alteração do metabolismo mineral e ósseo na DRC, estudos recentes sugerem que o aumento dos níveis do fator de crescimento de fibroblastos (FGF-23) precede a redução de calcitriol e pode ser o fator inicial que desencadeia o desenvolvimento do hiperparatireoidismo secundário. ${ }^{3}$ Os níveis séricos de FGF-23 aumentam precocemente na DRC, antes de qualquer alteração nos níveis de $\mathrm{Ca}, \mathrm{P}, \mathrm{PTH}$ ou calcitriol. FGF-23 é um hormônio fosfatúrico, produzido no osso, e níveis elevados deste hormônio resultam em perda renal de $\mathrm{P}$ e supressão de calcitriol. Os níveis de FGF-23 podem ser regulados pela ingestão de $\mathrm{P}$ e aumentam com a progressão da DRC, estando os valores muito elevados nos pacientes com DRC estágio V. Nos pacientes com DRC, os níveis de calcitriol e de FGF-23 se correlacionam negativamente, sugerindo um papel significativo do hormônio no metabolismo mineral, especificamente na redução dos níveis de 25-hidroxivitamina D associado à DRC. ${ }^{3}$ É provável que FGF-23 regule a glândula paratireoide, seja suprimindo a liberação de PTH, seja atuando diretamente na secreção e PTH por um mecanismo independente de suas ações no metabolismo de 25-hidroxivitamina D. ${ }^{1}$ Em pacientes com DRC, a redução dos níveis de 1,25-vit D mediada via FGF23 ocorre precocemente no curso da DRC, antes de qualquer alteração nas concentrações séricas de $\mathrm{Ca}$ e P e elevação nos níveis séricos de PTH. A redução dos níveis de calcitriol parece ser uma resposta adaptativa para limitar os efeitos tóxicos da hiperfosfatemia. Nos estágios mais avançados da DRC, a retenção de $\mathrm{P}$ e consequente hiperfosfatemia diretamente suprimem a atividade da $1 \alpha$ hidroxilase. ${ }^{1,4,5}$ O grupo de estudo recomenda avaliar o distúrbio bioquímico da DRC-DMO na criança a partir do estágio II da DRC (Tabela 1). ${ }^{2}$
O termo osteodistrofia renal (OR) está reservado para as alterações histológicas da doença óssea associada à DRC. A OR é classicamente dividida em doenças ósseas de alta e baixa remodelação. Dentre as doenças de alta remodelação encontrase o hiperparatireoidismo secundário (HPS), cuja manifestação óssea é a osteíte fibrosa, e a doença mista (DM). As doenças de baixa remodelação compreendem a doença óssea adinâmica (DOA) e a osteomalacia (OM). A biópsia óssea continua sendo o padrão-ouro para o diagnóstico da OR. Porém, por ser um método invasivo e restrito a alguns centros, a OR é comumente avaliada através de exames bioquímicos (Tabela 1).

$\mathrm{Na}$ criança, o HPS ocorre mais precocemente que no adulto, sendo já observado no estágio II da DRC. ${ }^{7}$ Além disso, a acidose metabólica per se contribui para o aumento da reabsorção óssea. Dessa forma, recomenda-se a determinação dos níveis séricos de $\mathrm{Ca}, \mathrm{P}$ e PTH a partir do estágio II da DRC, assim como do $\mathrm{pH}, \mathrm{HCO}_{3}$ ou $\mathrm{CO}_{2}$ total.

$\mathrm{Na}$ DRC estágio $\mathrm{V}$ ou dialítico, os níveis séricos de PTH entre 200 e 300 pg/mL refletem uma remodelação óssea próxima do normal (Tabela 2). Níveis acima ou abaixo desses valores são mais compatíveis com os diagnósticos de doença óssea de alta ou baixa remodelação, respectivamente. ${ }^{4,7,8}$ Para a FA devem ser considerados os valores normais fornecidos pelo laboratório, de acordo com a idade.

\section{Níveis séricos de $\mathrm{Ca}$ e $\mathrm{P}$ e produto $\mathrm{Ca} \times \mathrm{P}$}

2.1 Na DRC estágios I-IV os níveis séricos de Ca e P devem ser mantidos dentro dos limites da normalidade (Tabela 3) (Evidência).

2.2 Os níveis séricos de Ca total devem ser mantidos entre 8,8 e $9,7 \mathrm{mg} / \mathrm{dL}(2,20-2,37$ $\mathrm{mmol} / \mathrm{L})$, preferencialmente no limite inferior (Opinião).

\begin{tabular}{cccc} 
Tabela 3 & \multicolumn{2}{c}{$\begin{array}{c}\text { VALORES SÉRICOS NORMAIS DE CA TOTAL, } \\
\text { CA IÔNICO E P COM RELAÇÃO À IDADE }\end{array}$} \\
Idade & $\begin{array}{c}\text { Ca total } \\
\text { (mg/dL) }\end{array}$ & $\begin{array}{c}\text { Ca iônico } \\
(\mathrm{mmol} / \mathrm{L})\end{array}$ & $\mathrm{P}(\mathrm{mg} / \mathrm{dL})$ \\
\hline 0-11 meses & $8,8-11,3$ & $1,22-1,40$ & $4,8-7,4$ \\
1-5 anos & $9,4-10,8$ & $1,22-1,32$ & $4,5-6,5$ \\
6-12 anos & $9,4-10,3$ & $1,15-1,32$ & $3,6-5,8$ \\
13-20 anos & $8,8-10,2$ & $1,12-1,30$ & $2,3-4,5$ \\
\hline
\end{tabular}


2.3 Quando o nível sérico de Ca total for superior a $10,2 \mathrm{mg} / \mathrm{dL}(2,54 \mathrm{mmol} / \mathrm{L})$, ajustar o tratamento de acordo com as recomendações:

2.3.1 Descontinuar o uso de quelantes de P contendo Ca e considerar o uso de quelantes de $\mathrm{P}$ que não contenham Ca ou metal (Opinião).

2.3.2 Descontinuar o uso de vitamina $\mathrm{D}_{2}$ ou $\mathrm{D}_{3}$ até a normalização do nível sérico de Ca total (Opinião).

2.3.3 Caso o nível sérico de Ca total persista acima de 10,2 mg/dL (2,54 mmol/L), apesar das medidas terapêuticas contidas nas Diretrizes 2.3.1 e 2.3.2, reduzir a concentração de $\mathrm{Ca}$ da solução de diálise (Opinião).

2.4 A dose máxima de Ca elementar fornecida pelo quelante de $\mathrm{P}$ e pelo $\mathrm{Ca}$ dietético não deve exceder em 2 vezes a recomendação da ingestão dietética de Ca para a idade (Tabela 4) (Opinião). A ingestão total de Ca elementar (incluindo o Ca dietético) não deve exce$\operatorname{der} 2,5 \mathrm{~g} / \mathrm{dia}$ (Opinião).

\begin{tabular}{c|cc}
\hline Tabela 4 & $\begin{array}{c}\text { INGESTÃO DIÁRIA RECOMENDADA (RDI) } \\
\text { PARA O CA }\end{array}$ \\
\hline Idade (anos) & $\begin{array}{c}\text { Ingestão adequada } \\
\text { (mg/dia) }\end{array}$ & $\begin{array}{c}\text { Níveis superiores } \\
\text { toleráveis (g/dia) }\end{array}$ \\
\hline $0-0,5$ & 210 & $\mathrm{ND}$ \\
$0,5-1,0$ & 270 & $\mathrm{ND}$ \\
$1-3$ & 500 & 2,5 \\
$4-8$ & 800 & 2,5 \\
$9-13$ & 1.300 & 2,5 \\
$14-18$ & 1.300 & 2,5 \\
\hline
\end{tabular}

ND - Não determinado

2.5 O produto $\mathrm{Ca} \times \mathrm{P}$ deve ser mantido abaixo de $55 \mathrm{mg}^{2} / \mathrm{dL}^{2}$ em adolescentes acima de 12 anos e abaixo de $65 \mathrm{mg}^{2} / \mathrm{dL}^{2}$ em crianças mais jovens (Opinião).

2.6 Níveis séricos de Ca total abaixo do limite inferior de normalidade (menor que $8,8 \mathrm{mg} / \mathrm{dL}$ ou 2,20 mmol/L) devem ser corrigidos (Opinião).

2.6.1 O tratamento da hipocalcemia $(<8,8 \mathrm{mg} / \mathrm{dL}$ ou 2,20 $\mathrm{mmol} / \mathrm{L}$ ) inclui a administração oral de sais de $\mathrm{Ca}$, tais como carbonato, acetato ou gluconato de $\mathrm{Ca}$, administrados em horários distantes das refeições, e/ou administração oral de vitamina $\mathrm{D}_{2}$ ou $\mathrm{D}_{3}$ (Evidência).
2.7 Na DRC estágios I a IV os níveis de P devem ser mantidos dentro dos limites da normalidade para idade (Tabela 3) (Opinião), de preferência nos limites inferiores de normalidade (Evidência).

2.8 Na DRC estágio V ou dialítico, os níveis de $\mathrm{P}$ séricos devem ser mantidos entre 4-6 mg/dL na criança com idade de 1 a 12 anos e entre 3,5- 5,5 mg/dL (1,13-1,78 mmol/L) no adolescente (Evidência).

2.9 Em crianças portadoras de tubulopatias perdedoras de P (cistinose, síndrome de Fanconi ou outras causas de hipofosfatemia), a hipofosfatemia deve ser corrigida com dieta, suplementação oral de $\mathrm{P}$ ou redução na dose dos quelantes (Evidência).

\section{RACIONAL}

A determinação do Ca iônico deve ser utilizada preferencialmente. Os níveis de Ca total, quando utilizados, devem ser corrigidos pela albumina sérica, ${ }^{9}$ de acordo com a fórmula: Ca total corrigido $(\mathrm{mg} / \mathrm{dL})$ = concentração de $\mathrm{Ca}(\mathrm{mg} / \mathrm{dL})+0,8 \times[4$ - concentração sérica de albumina $(\mathrm{g} / \mathrm{dL})]$.

A hipocalcemia deve ser corrigida, pois favorece o desenvolvimento do HPS, interfere na mineralização óssea e está associada à maior mortalidade. Da mesma forma, a hipercalcemia, secundária ao excesso de ingestão de $\mathrm{Ca}$ ou uso inadequado de vitamina $\mathrm{D}_{2}$ ou $\mathrm{D}_{3}$, deve ser evitada, pois também está associada à maior mortalidade.

Embora a recomendação dietética de Ca (RDI, $2000)^{10}$ não tenha ainda sido estabelecida, na Tabela 4 é apresentada a ingestão de Ca adequada e seu limite superior na criança e no adolescente. A opinião do grupo de trabalho do KDOQI é a recomendação de uma ingestão de Ca de 2 vezes a cota da RDI para a idade (máximo de 2,5 g/dia) considerando dieta e suplementos, como a cota apropriada para crianças e adolescentes com DRC (Opinião). Em pacientes em diálise, a suplementação de Ca de $3 \mathrm{~g} /$ dia em adição a 400-500 mg de Ca dietético resulta em hipercalcemia em 36\% dos pacientes. ${ }^{11-13}$ Pacientes com DRC em tratamento com metabólitos da 25-hidroxivitamina D ou suplementos de Ca têm maior risco de desenvolver hipercalcemia, especialmente aqueles pacientes com doença óssea de baixa remodelação. ${ }^{14,15}$ Hipercalcemia mais hiperfosfatemia resultam em um produto $\mathrm{Ca}$ x $\mathrm{P}$ elevado e risco de calcificação de 
tecidos moles. Nessa situação, a restrição dietética de P é aconselhável. ${ }^{16,17}$

Os sais de Ca são bem tolerados e devem ser usados em doses que não sejam superiores àquelas da RDI. Os principais sais de Ca são: gluconato (9\% de Ca elementar); lactato (13\% de Ca elementar); acetato (25\% de Ca elementar) e carbonato de $\mathrm{Ca}(40 \%$ de Ca elementar). Os demais compostos não devem ser utilizados. Os sais de $\mathrm{Ca}$ administrados como quelante de $\mathrm{P}$ devem ser tomados junto com as refeições e, longe delas, quando o objetivo for a suplementação de Ca.

$\mathrm{Na}$ criança, existem variações importantes dos níveis de $\mathrm{P}$ sérico, dependendo da idade, atingindo os mesmos níveis de adultos na adolescência (Tabela 3). ${ }^{18,19}$ Estudos sugerem que a manutenção de níveis séricos normais de $\mathrm{P}$ na DRC é crucial na prevenção do HPS e da DCV. ${ }^{20,21}$ Níveis de $\mathrm{P}$ acima de $7 \mathrm{mg} / \mathrm{dL}$ e abaixo de 2,5 $\mathrm{mg} / \mathrm{dL}$ aumentam significativamente a mortalidade. Na DRC, a retenção de P é proporcional à perda da filtração glomerular, e as concentrações de $\mathrm{P}$ variam de acordo com os estágios da DRC. Dessa forma, recomenda-se manter os níveis de P dentro da normalidade na DRC visando prevenir o HPS e reduzir as taxas de morbidade e mortalidade. ${ }^{17}$

\section{Controle dietético do P na DRC}

A ingestão de $\mathrm{P}$ deve seguir as recomendações do K/DOQI sobre as diretrizes da nutrição pediátrica de $100 \%$ da RDI para idade (Tabela 5), em crianças no estágio III a V com os níveis séricos de PTH acima dos valores esperados para o estágio da DRC e os níveis séricos de $\mathrm{P}$ dentro dos limites da normalidade para idade (Tabela 3) (Evidência).

A ingestão de P deve ser reduzida em $80 \%$ da RDI (Tabela 5), quando os níveis séricos de PTH estiverem

\begin{tabular}{ccc} 
Tabela 5 & \multicolumn{2}{l}{$\begin{array}{l}\text { INGESTÃO DIÁRIA RECOMENDADA (RDI) } \\
\text { NORMAL E REDUZIDA DE FÓSFORO PARA } \\
\text { A IDADE }\end{array}$} \\
\hline Idade (anos) & P dietético (RDI) & $80 \% \mathrm{da} \mathrm{RDI}$ \\
\hline $0-0,5$ & $100 \mathrm{mg} / \mathrm{dia}$ & $80 \mathrm{mg} / \mathrm{dia}$ \\
$0,5-1,0$ & $275 \mathrm{mg} / \mathrm{dia}$ & $220 \mathrm{mg} / \mathrm{dia}$ \\
$1-3$ & $460 \mathrm{mg} / \mathrm{dia}$ & $368 \mathrm{mg} / \mathrm{dia}$ \\
$4-8$ & $500 \mathrm{mg} / \mathrm{dia}$ & $400 \mathrm{mg} / \mathrm{dia}$ \\
$9-18$ & $1.250 \mathrm{mg} / \mathrm{dia}$ & $1000 \mathrm{mg} / \mathrm{dia}$ \\
\hline
\end{tabular}

RDI recomendação dietética para a idade acima dos valores esperados para o estágio da DRC e a concentração sérica de $\mathrm{P}$ estiver dentro dos limites de normalidade para idade (Tabela 3) (Evidência).

Após o início da restrição dietética de P, monitorar o P sérico a cada 3 meses nos estágios III e IV da DRC e mensalmente, no estágio $\mathrm{V}$, devendo-se evitar níveis de $\mathrm{P}$ sérico abaixo dos valores normais para idade (Opinião).

\section{RACIONAL}

A retenção de $\mathrm{P}$ ocorre em estágios precoces da doença renal crônica da criança (estágio II) e tem um papel importante para o desenvolvimento do HPS. A redução da produção de calcitriol no rim reduz o efeito inibitório do calcitriol sobre a liberação do PTH com consequente hiperparatireoidismo, que aumenta a excreção urinária de P. Portanto, nos estágios iniciais da DRC, os níveis de $\mathrm{P}$ sérico estão normais. A adaptação não é completa, e ocorrem pequenos aumentos dos níveis séricos de $\mathrm{P}$ que se tornam mais elevados com a progressão da DRC; a hiperfosfatemia eleva ainda mais os níveis de PTH pela supressão da produção de calcitriol e pelo efeito direto na glândula paratireoide. ${ }^{22}$ Mesmo nos estágios iniciais da DRC, a restrição dietética de $\mathrm{P}$ reduz os níveis de PTH e aumenta os níveis de 1,25-vit D ou calcitriol. ${ }^{23} \mathrm{~A}$ diminuição da ingestão do $\mathrm{P}$ contribui para o controle do HPS, sendo a primeira etapa no tratamento desta alteração. A elevação dos níveis séricos de $\mathrm{P}$ está associada à progressão da DRC, e seu controle tem impacto positivo na evolução da DRC. ${ }^{24,25}$ Portanto, nos estágios II e III da DRC o controle do HPS pode ser obtido com a redução da ingestão de P. A opinião do grupo de trabalho do KDOQI ${ }^{4}$ é de se reduzir a ingestão de $\mathrm{P}$ de acordo com os valores da RDI (Tabela 5), mesmo quando os níveis séricos de $\mathrm{P}$ estiverem dentro dos valores normais. Nos estágios IV e V, quando os níveis séricos de $\mathrm{P}$ estiverem elevados, a RDI deve ser reduzida em $80 \%$ (Tabela 5). ${ }^{17}$ Concentração sérica de $\mathrm{P}$ abaixo do valor de normalidade deve ser evitada devido ao risco potencial de comprometimento do crescimento linear pela hipofosfatemia.

\section{Uso de quelantes de P na DRC}

\section{DRC estágio II a IV}

4.1 Os quelantes de $\mathrm{P}$ devem ser prescritos na DRC estágios II a V quando houver hiperfosfatemia, apesar da restrição dietética de $\mathrm{P}$ (Opinião). 
4.2 Na DRC estágio II a IV os quelantes de P contendo Ca são efetivos para reduzir os níveis séricos de $\mathrm{P}$ e devem ser usados como terapia inicial (Evidência).

\section{DRC V}

4.3 Na DRC estágio V os quelantes de P contendo $\mathrm{Ca}$, assim como os quelantes de $\mathrm{P}$ sem metal, são efetivos para reduzir os níveis séricos de $\mathrm{P}$ (Evidência). Os quelantes de $\mathrm{P}$ contendo $\mathrm{Ca}$ devem ser usados inicialmente em lactentes e crianças jovens. Para as crianças mais velhas pode ser administrado qualquer dos quelantes citados (Opinião).

\begin{tabular}{ccc} 
Tabela 6 & $\begin{array}{l}\text { Dose INICIAL Do QUELANTE À BASE DE } \\
\text { CA }\end{array}$ \\
Idade & $\begin{array}{l}\text { Dose do quelante } \\
\text { de P contendo Ca }\end{array}$ & Posologia \\
\hline $0-1$ ano & $250 \mathrm{mg}$ & 3 a 5 vezes ao dia \\
$1-4$ anos & $500 \mathrm{mg}$ & 2 a 3 vezes ao dia \\
$5-8$ anos & $500 \mathrm{mg}$ & 3 a 4 vezes ao dia \\
$9-19$ anos & $500 \mathrm{mg}$ & 5 vezes ao dia \\
\hline
\end{tabular}

4.4 Em pacientes em diálise que permanecem com hiperfosfatemia (acima do limite superior de normalidade) apesar do uso de quelantes de $\mathrm{P}$ contendo $\mathrm{Ca}$ ou outro quelante de $\mathrm{P}$ sem $\mathrm{Ca}$ e sem metal, a prescrição da diálise deve ser modificada para controlar a hiperfosfatemia (Opinião).

4.5 A dose total de Ca elementar proveniente do quelante contendo Ca não deve exceder duas vezes a RDI para o $\mathrm{Ca}$, baseada na idade (Opinião), e a ingestão total de Ca elemento incluindo o teor de $\mathrm{Ca}$ dietético não deve exceder 2,5 g/dia, Tabela 4 (Opinião).

4.6 A dose de quelantes de P contendo Ca deve ser reduzida em pacientes com níveis séricos corrigidos de $\mathrm{Ca}>10,2 \mathrm{mg} / \mathrm{dL}(2,54 \mathrm{mmol} / \mathrm{L})$ ou com níveis de $\mathrm{PTH}<150$ pg/mL (150 ng/L) em duas medidas consecutivas (Evidência).

4.7 Em adolescentes com níveis séricos de $\mathrm{P}>$ 7,0 $\mathrm{mg} / \mathrm{dL}$ (2,26 $\mathrm{mmol} / \mathrm{L})$, pode ser usado quelante contendo alumínio $(\mathrm{Al})$ por curto período de tempo (4 a 6 semanas) e apenas uma vez, sendo substituído após este período por outro quelante de P (Evidência). Em lactentes seu uso está contraindicado (Opinião).
4.8 Nas crianças recebendo quelante de P contendo alumínio deve-se evitar o uso concomitante de produtos contendo citrato pelo risco de aumento da absorção de $\mathrm{Al}$ induzida pelo citrato (Evidência).

4.9 Quelantes de P sem Ca ou metal (cloridrato de sevelamer) devem ser utilizados com critério em crianças. Estão indicados em crianças cuja hiperfosfatemia persiste após o uso de quelantes contendo $\mathrm{Ca}$ e adequação da diálise. O cloridrato de sevelamer está indicado em todas as crianças que apresentem Ca sérico acima dos limites superiores para a idade (Tabela 3) em todos os estágios da DRC (Opinião).

4.10 Pacientes em diálise que permaneçam hiperfosfatêmicos, apesar do uso de quelantes de $\mathrm{P}$, devem ter suas prescrições de diálise modificadas, visando o melhor controle do $\mathrm{P}$ (Opinião).

4.10.1 Nos pacientes em diálise peritoneal (DP), o volume da solução deve ser aumentado para 1000 - $1400 \mathrm{~mL} / \mathrm{m}^{2}$ de superfície corporal, além do aumento do tempo de permanência e/ou o acréscimo de trocas de banho (Evidência).

4.10.2 Nos pacientes em hemodiálise (HD) deve-se aumentar a frequência das sessões e/ou prolongar o tempo de diálise, podendo ser utilizada a diálise diária diurna ou noturna (Evidência).

4.11 A dose do quelante de $\mathrm{P}$ à base de $\mathrm{Ca}$ deve ser diminuída nos pacientes em HD quando o Ca sérico for superior ou igual a $10,2 \mathrm{mg} /$ $\mathrm{dL}$ (2,54 mmol/L) ou PTH a $150 \mathrm{pg} / \mathrm{mL}$ em duas medidas consecutivas (Opinião).

\section{RACIONAL}

A adesão da criança à restrição dietética de $\mathrm{P}$ é baixa, uma vez que a maioria dos alimentos preferidos pelas crianças é rica em P. Portanto, apesar da tentativa de restrição dietética de $\mathrm{P}$ o uso de quelantes de $\mathrm{P}$ se faz necessário para reduzir a absorção intestinal desse elemento pela formação no trato gastrointestinal de complexos com P que são pouco solúveis. Deve ser iniciado quando o nível de P se mantém alto apesar da restrição dietética ou quando a restrição dietética compromete a ingestão de outros nutrientes essenciais. Recomenda-se iniciar o tratamento da hiperfosfatemia com quelantes de $\mathrm{P}$ contendo $\mathrm{Ca}$, sendo 
os mais utilizados o carbonato ou o acetato de $\mathrm{Ca}$. $\mathrm{O}$ carbonato de $\mathrm{Ca}$ foi o primeiro composto de $\mathrm{Ca}$ usado, e os estudos mostram sua eficácia no controle dos níveis séricos de P. ${ }^{4,5}$ Os quelantes de P contendo Ca geralmente são iniciados na dose de $500 \mathrm{mg}$ por $200 \mathrm{mg}$ de $\mathrm{P}$ dietético. ${ }^{26}$ Um alternativa é iniciar com a dose de $50 \mathrm{mg} / \mathrm{kg} / \mathrm{dia}$, dose mais baixa do que a utilizada nos estudos clínicos. ${ }^{26} \mathrm{Em}$ geral, a dose inicial para carbonato de Ca e acetato de Ca é 600-1500 mg e 375-750 mg de Ca elemento por dia, respectivamente, dose que deve ser ajustada até a normalização dos níveis séricos de P. ${ }^{1} \mathrm{O}$ uso de altas dose de quelantes contendo $\mathrm{Ca}$ tem sido associado ao desenvolvimento de CV em adultos e crianças em diálise, assim como adultos no estágio IV da DRC. ${ }^{1}$ Os quelantes de P devem ser ingeridos 10 a 15 minutos antes ou durante a refeição, visando aumentar a ação quelante e a excreção de $\mathrm{P}$ nas fezes. A Tabela 7 apresenta $\mathrm{O}$ percentual de absorção de $\mathrm{Ca}$, assim como os efeitos colaterais dos principais quelantes de $\mathrm{P}$. A ação quelante é limitada: 1 grama de carbonato de Ca quela $39 \mathrm{mg}$ de P, 1 grama de acetato de Ca se liga a 45 mg de P e $400 \mathrm{mg}$ de sevelamer quela $32 \mathrm{mg}$ do íon. Portanto, esses quelantes só serão efetivos se houver restrição dietética de P. ${ }^{4}$ É importante lembrar que a aderência ao tratamento com quelantes de P é difícil, sendo necessário o controle regular e a identificação da melhor maneira de se oferecer a medicação para a criança. Para os lactentes, o carbonato de Ca pode ser oferecido na forma de pó ou solução oral a $10 \%$. $\mathrm{O}$ efeito colateral mais frequente dos quelantes de $\mathrm{P}$ à base de $\mathrm{Ca}$, principalmente quando associado ao calcitriol, é a hipercalcemia $(\mathrm{Ca}>10,2 \mathrm{mg} / \mathrm{dL}$ ou 2,54 $\mathrm{mmol} / \mathrm{L})$; a dose total de Ca elemento não deve ser superior a duas vezes a RDI para a idade, atingindo o máximo de $2.500 \mathrm{mg} / \mathrm{dia}$, incluindo o teor de $\mathrm{Ca}$ da dieta. Nesses casos, recomenda-se utilizar quelantes sem $\mathrm{Ca}$ ou metal, como o cloridrato de sevelamer (Renagel®). 2,4,27

O cloridrato de sevelamer é um polímero sintético que não contém $\mathrm{Al}$ ou $\mathrm{Ca}$ na sua composição, é resistente à degradação digestiva e não é absorvido pelo trato gastrointestinal. Os comprimidos podem ser dissolvidos em $5 \mathrm{~mL}$ de água e administrados por via oral ou enteral. O uso do cloridrato de sevelamer em crianças tem se mostrado seguro e eficaz, embora dados sobre o uso deste agente na criança são limitados. ${ }^{28} \mathrm{O}$ uso de sevelamer por 8 semanas em um estudo randomizado mostrou que o quelante foi tão eficaz quanto o acetato de Ca para reduzir os níveis séricos de P. ${ }^{29}$ Sevelamer foi associado à redução nos níveis de colesterol, LDL-colesterol e da frequência dos episódios hipercalcêmicos, mas com aumento da acidose metabólica (Tabela 7). Um estudo-piloto para avaliar a eficácia de hidrocloreto de sevelamer como quelante de $\mathrm{P}$ em crianças em diálise usou uma dose inicial de $121 \pm 50 \mathrm{mg} / \mathrm{kg}(4,5 \pm 5 \mathrm{~g} / \mathrm{dia})$ e final de $163 \pm 46 \mathrm{mg} / \mathrm{kg}(6,7 \pm 2,4 \mathrm{~g} / \mathrm{dia}) .{ }^{30} \mathrm{O}$ medicamento foi bem tolerado, com redução dos níveis de $\mathrm{P}$ sérico, cujos valores permaneceram normais na maioria dos pacientes do estudo. Toda atenção deve ser dada ao fato de que o cloridrato de sevelamer pode agravar a acidose metabólica em crianças. Uma nova formulação - carbonato de sevelamer (ainda não existente em nosso meio) favoreceria a correção da acidose metabólica. Em crianças em diálise, o carbonato de sevelamer foi efetivo como quelante de $\mathrm{P}$ comparado ao cloreto de sevelamer, ocorrendo aumento dos níveis séricos de bicarbonato que permitiram a suspensão da terapia com bicarbonato. ${ }^{31}$

$\mathrm{O}$ controle dos níveis séricos de $\mathrm{P}$ é mais difícil no paciente em diálise, e a meta é manter os níveis de $\mathrm{P}$ pouco abaixo do percentil 50 para a idade. A remoção de P pela diálise peritoneal é de 300 a 400 mg/ dia e pela HD de $800 \mathrm{mg} / \mathrm{sessão,} \mathrm{valores} \mathrm{insuficientes}$ para manter normais os níveis séricos de P. Portanto, o uso de quelantes de P é sempre necessário nos pacientes em diálise. É essencial monitorar os níveis séricos de $\mathrm{P}$ para prevenir hipofosfatemia, que é consequência da restrição dietética mais severa e uso de doses altas dos quelantes. Essa recomendação é de particular importância no lactente devido à ingestão baixa, dose alta do quelante e maior remoção peritoneal de $\mathrm{P}$ pela maior área de superfície peritoneal. ${ }^{5}$

Tabela 7 Percentual de absorção de Ca e efeitos colaterais dos ouelantes de P

\begin{tabular}{|c|c|c|}
\hline Componente & Porção estimada de $\mathrm{Ca}$ absorvido & Efeitos colaterais possíveis \\
\hline Carbonato de $\mathrm{Ca}$ & $20 \%$ a $30 \%$ & $\begin{array}{l}\text { Hipercalcemia, calcificação extraesquelética, } \\
\text { sintomas GI }\end{array}$ \\
\hline Acetato de $\mathrm{Ca}$ & $\begin{array}{c}\text { Com alimento } 21 \% \\
\text { Entre as refeições 40\% }\end{array}$ & $\begin{array}{l}\text { Hipercalcemia, calcifição extraesquelética, } \\
\text { sintomas Gl }\end{array}$ \\
\hline Cloridrato de sevelamer & Nenhuma & Sintomas GI, acidose metabólica \\
\hline
\end{tabular}


$\mathrm{O}$ uso de quelantes contendo $\mathrm{Al}$ deve ser restrito ao tratamento da hiperfosfatemia grave (> $7 \mathrm{mg} / \mathrm{dL}$ ) no adolescente, associada com hipercalcemia ou produto $\mathrm{Ca}$ x $\mathrm{P}$ elevado, condições agravadas pelo uso de quelantes contendo $\mathrm{Ca}$. A dose de hidróxido de Al não deve exceder $30 \mathrm{mg} / \mathrm{kg} /$ dia e deve administrado por um período não superior a 4-6 semanas. Os níveis plasmáticos de $\mathrm{Al}$ devem ser monitorados, e o uso de compostos contendo citrato devem ser evitados, porque citrato aumento a absorção intestinal de $\mathrm{Al}$ e aumenta o risco de intoxicação. ${ }^{1,27}$

5 Prevenção e tratamento da deficiência de 25-hidroxivitamina $\mathrm{D}$ em crianças com DRC

Estágio II - IV

5.1 Nível sérico de PTH elevado para o estágio da DRC (Diretrizes 1 e 2, Tabelas 2 e 3), monitorar os níveis de vitamina D [25-vit D] (Evidência). As medidas devem ser repetidas se forem feitas modificações dietéticas ou no estilo de vida.

5.2 Nível sérico de 25 -vit D menor que $30 \mathrm{ng} / \mathrm{mL}$, iniciar suplementação com vitamina $\mathrm{D}_{2}$ ou $\mathrm{D}_{3}$ (ergocalciferol ou colecalciferol, Tabelas 8 e 9) (Opinião).

5.3 Após iniciar suplementação com vitamina $\mathrm{D}_{2}$ ou $\mathrm{D}_{3}$ :
5.3.1 Monitorar os níveis séricos de $\mathrm{Ca}$ e $\mathrm{P}$ após 1 mês e a seguir a cada 3 meses (Opinião).

5.3.2 Descontinuar o uso de vitamina $\mathrm{D}_{2}$ ou $\mathrm{D}_{3}$ se Ca total maior que $10,2 \mathrm{mg} / \mathrm{dL}(2,54 \mathrm{mmol} / \mathrm{L})$ (Opinião).

5.3.3 Se os níveis séricos de Ca total forem superiores a $10,2 \mathrm{mg} / \mathrm{dL}(2,54 \mathrm{mmol} / \mathrm{L})$, suspender o uso de vitamina $\mathrm{D}_{2}$ ou $\mathrm{D}_{3}$ e todas as formas de vitamina (Opinião).

5.3.4 Se os níveis séricos de P se elevarem acima do valor normal para a idade (Tabela 3 ), iniciar restrição dietética de $\mathrm{P}$ (diretrizes 2 e 3), ou se a hiperfosfatemia persisitir e os níveis séricos de 25 -vit $\mathrm{D}$ forem $<30 \mathrm{ng} / \mathrm{mL}$, iniciar quelante oral de P. Se os níveis de 25 -vit D estiverem normais, suspender o uso da vitamina (Opinião).

5.3.5 Após reposição com vitamina $\mathrm{D}_{2}$ ou $\mathrm{D}_{3}$, manter suplementação com um preparado multivitamínico contendo 25-hidroxivitamina $\mathrm{D}$ e proceder à dosagem anual dos níveis séricos (Opinião).

\section{DRC estágio $\mathrm{V}$}

5.4 Tratamento com calcitriol na DRC estágio V deve ser iniciado quando os níveis de PTH forem maiores que $300 \mathrm{pg} / \mathrm{mL}$ (Opinião).

Tabela 8 SuPLEMENTAÇÃO DE VITAMINA $D_{2}$ OU $D_{3}$ NA DRC ESTÁGIOS II-IV

\begin{tabular}{|c|c|c|c|c|}
\hline $\begin{array}{l}\text { Nível sérico de } \\
25-\text { vit } D(\mathrm{ng} / \mathrm{mL})\end{array}$ & $\begin{array}{c}\text { Grau de } \\
\text { deficiência }\end{array}$ & Dose de 25-vit D (oral) & $\begin{array}{l}\text { Duração } \\
\text { (meses) }\end{array}$ & Controle do nível sérico \\
\hline$<5$ & Severo & $\begin{array}{l}8.000 \text { UI/dia/4 sem. ou } 50.000 \text { UI/ } \\
\text { sem/4 sem. Após, } 4.000 \text { UI/dia/2 } \\
\text { meses ou } 50.000 \text { UI 2x/mês/2 meses }\end{array}$ & 3 meses & $\begin{array}{l}\text { Medir nível de } 25 \text {-vit D } \\
\text { após } 3 \text { meses }\end{array}$ \\
\hline $5-15$ & Moderado & $\begin{array}{c}4.000 \text { UI/dia/3 meses ou } 50.000 \text { UI 2x/ } \\
\text { mês/3 meses }\end{array}$ & 3 meses & $\begin{array}{l}\text { Medir nível de 25-vit D } \\
\text { após } 3 \text { meses }\end{array}$ \\
\hline $16-30$ & Insuficiente & 2.000 UI/dia ou 50.000 UI/mês & 3 meses & $\begin{array}{l}\text { Medir nível de 25-vit D } \\
\text { após } 3 \text { meses }\end{array}$ \\
\hline
\end{tabular}

$\mathrm{UI}=$ unidades internacionais; sem. = semanas

Tabela 9 SuPLEMENTAÇÃO DE VITAMINA $D_{2}$ OU $D_{3}$ NA DRC ESTÁGIO V

\begin{tabular}{|c|c|c|c|c|}
\hline $\begin{array}{l}\text { Nível sérico } 25 \text {-vit D } \\
(\mathrm{ng} / \mathrm{mL})\end{array}$ & $\begin{array}{l}\text { Grau de } \\
\text { deficiência }\end{array}$ & Dose de 25-hidroxivitamina D (oral) & Duração & Controle do nível sérico \\
\hline$<5$ & Severo & $\begin{array}{c}8.000 \mathrm{UI} / \mathrm{dia} / 4 \mathrm{sem} \text {. ou } 50.000 \mathrm{UI} / \\
\text { sem/4 sem. Após, } 4.000 \mathrm{UI} / \mathrm{dia} / 2 \\
\text { meses ou } 50.000 \mathrm{UI} 2 \times / \mathrm{mês} / 2 \text { meses }\end{array}$ & 3 meses & Mensal \\
\hline $5-15$ & Moderado & $\begin{array}{c}4.000 \mathrm{UI} / \mathrm{dia} / 12 \mathrm{sem} \text {. ou } 50.000 \mathrm{UI} / 2 \mathrm{x} / \\
\mathrm{mês} / 3 \text { meses }\end{array}$ & 3 meses & Mensal \\
\hline $16-30$ & Insuficiente & $2.000 \mathrm{IU} /$ dia ou $50.000 \mathrm{UI} / \mathrm{mês}$ & 3 meses & Mensal \\
\hline
\end{tabular}




\section{RACIONAL}

A 25-hidroxivitamina D é fundamental para a homeostase do $\mathrm{Ca}, \mathrm{P}$ e PTH, tendo importante papel no crescimento da criança. Os níveis de 25 -vit D refletem o estoque corporal da 25-hidroxivitamina D. A principal causa de deficiência de 25-hidroxivitamina D em portadores de DRC é nutricional, com estimativas alarmantes de uma incidência de $70 \%$ entre jovens americanos. $^{32}$ Em países como o nosso, com elevados índices de desnutrição e com predomínio da população de raça negra, estima-se que a deficiência de 25-hidroxivitamina D também seja elevada. Estudo realizado no Recife, por Linhares e cols., ${ }^{33}$ comparando níveis de 25-hidroxivitamina D em 412 crianças saudáveis e 226 desnutridas, não demonstrou diferença entre os dois grupos. Além disso, os níveis de 25-hidroxivitamina $\mathrm{D}$ encontrados foram superiores aos de crianças europeias, o que foi atribuído à elevada exposição ao sol. ${ }^{34}$ Estudos mais recentes em diferentes países mostram deficiência de 25-hidroxivitamina D em crianças aparentemente saudáveis com incidência variando entre $10 \%$ a $50 \%$, desde o período neonatal até a adolescência, sendo mais frequente em crianças com baixo poder aquisitivo, adolescentes e naquelas de origem latina e africana. ${ }^{35}$

Outros fatores, como a proteinúria, contribuem para diminuição da 25-hidroxivitamina $\mathrm{D}$ na doença renal, devido à perda da proteína carreadora dessa vitamina. Estudos com 258 pacientes (idade média de 12,3 anos) portadores de DRC em todos os estágios, mas principalmente estágios I e II, mostraram valores de 25 -vit $\mathrm{D}$ menores que $30 \mathrm{ng} / \mathrm{mL}$ em mais de $60 \%$ dos pacientes com TFG estimada de $106 \pm$ $51 \mathrm{~mL} / \mathrm{min} / 1,73 \mathrm{~m}^{2}$, justificando sua suplementação na DRC. ${ }^{36}$ Por essa razão, o tratamento com 25-hidroxivitamina $\mathrm{D}$ deve ser iniciado precocemente para prevenir o desenvolvimento do HPS e o retardo do crescimento. Deficiência de 25-hidroxivitamina D na criança pode causar raquitismo. A 25-hidroxivitamina D regula mais de 200 genes, incluindo aqueles responsáveis pela regulação da proliferação celular. Possui ação no cérebro, mamas, cólon e células do sistema imune. Alguns destes tecidos expressam a enzima 1-alfa hidroxilase, responsável pela hidroxilação in situ da 25 -vit D, transformando-a em calcitriol. ${ }^{37}$

\section{Tratamento com calcitriol na DRC}

\section{DRC estágios II a IV}

6.1 Para os pacientes com DRC estágios II-IV, a reposição de calcitriol deve ser iniciada quando os níveis séricos de 25 -vit $\mathrm{D}$ forem maiores que $30 \mathrm{ng} / \mathrm{mL}$ (75 nmol/L) e PTH acima do esperado para os estágios de DRC (Diretriz 1, Tabela 2) (Evidência).

6.1.1 O calcitriol só deve ser administrado se o nível sérico de Ca total for menor que $10 \mathrm{mg} /$ $\mathrm{dL}(2,37 \mathrm{mmol} / \mathrm{L})$ e de $\mathrm{P}$ menor que o limite superior para a idade (Tabela 10) (Opinião).

6.2 Para pacientes em uso de calcitriol, o controle de $\mathrm{Ca}$ e $\mathrm{P}$ deve ser mensal nos primeiros 3 meses e depois trimestral; PTH sérico deve ser medido a cada 3 meses (Tabela 1) (Opinião).

6.3 A dose de calcitriol deve ser ajustada de acordo com as seguintes recomendações:

6.3.1 PTH abaixo dos limites normais para o estágio da DRC (Tabela 2), descontinuar temporariamente o calcitriol até elevação dos níveis de PTH para acima do valor-alvo (Opinião). Reiniciar tratamento com metade da dose anterior. Para doses menores que $0,25 \mu \mathrm{g} / \mathrm{dia}$ ou $0,05 \mu \mathrm{g}$ na forma líquida, prescrever o calcitriol em dias alternados (Opinião).

6.3.2 Ca sérico total maior que 10,2 mg/dL (2,37 $\mathrm{mmol} / \mathrm{L}$ ), suspender tratamento com calcitriol até que o seus níveis sejam inferiores a 9,8 $\mathrm{mg} / \mathrm{dL}(2,7 \mathrm{mmol} / \mathrm{L})$. O tratamento deve ser reiniciado com metade da dose anterior. Se a dose é inferior a uma cápsula de $0,25 \mu \mathrm{g}$ ou $0,05 \mu \mathrm{g}$ na forma líquida, prescrever o calcitriol em dias alternados (Opinião).

6.3.3 P sérico acima dos limites esperados para a idade (Tabela 3), suspender o calcitriol, iniciar

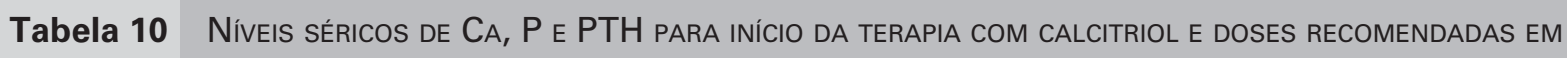
PACIENTES COM DRC ESTÁGIOS II A IV

\begin{tabular}{|c|c|c|c|}
\hline PTH (pg/mL) & Ca total $(\mathrm{mg} / \mathrm{dL})$ & $P(\mathrm{mg} / \mathrm{dL})$ & Dose calcitriol oral \\
\hline $\begin{array}{l}>70(\text { DRC } 2-3) \\
>110(\text { DRC } 4)\end{array}$ & $<10$ & $\leq$ nível recomendado para a idade & $\begin{array}{c}<10 \mathrm{~kg}: 0,05 \mu \mathrm{g} \text { dias alternados } \\
10-20 \mathrm{~kg}: 0,1-0,15 \mu \mathrm{g} / \mathrm{dia} \\
>20 \mathrm{~kg}: 0,25 \mu \mathrm{g} / \mathrm{dia}\end{array}$ \\
\hline
\end{tabular}


quelantes de $\mathrm{P}$ ou aumentar a dose do quelante já utilizado até a normalização dos níveis de P. Após normalização do $\mathrm{P}$, reiniciar calcitriol usando metade da dose anterior (Opinião).

6.4 Aumentar a dose de calcitriol em 50\% da dose inicial se não houver redução do PTH de pelo menos $30 \%$ em 3 meses após início do tratamento, desde que os níveis de Ca e P estejam normais (Opinião). Os níveis séricos de PTH, $\mathrm{Ca}$ e $\mathrm{P}$ devem ser dosados mensalmente por 3 meses.

\section{DRC Estágio V}

6.5 Para pacientes com DRC estágio V, com nível sérico de PTH maior ou igual a $300 \mathrm{pg} / \mathrm{mL}$, o calcitriol deve ser iniciado visando à redução dos níveis de PTH para valores entre 200-300 $\mathrm{pg} / \mathrm{mL}$ (Tabela 11) (Evidência).

6.6 Administração intermitente do calcitriol por via oral ou intravenosa é mais efetiva para baixar o PTH que doses diárias (Evidência).

6.7 Quando a terapia com calcitriol for iniciada ou modificada, a dosagem dos níveis séricos de $\mathrm{Ca}$ e $\mathrm{P}$ deve ser mensal por 3 meses e, posteriormente, a cada 3 meses. A dosagem do PTH deve ser mensal por 3 meses e trimestral após atingir os níveis-alvo de PTH (Opinião).

6.8 Para pacientes em DP a dose oral inicial de calcitriol $(0,5-1,0 \mu \mathrm{g})$ pode ser administrada 3 vezes/semana. Alternativamente, uma dose mais baixa $(0,25 \mu \mathrm{g})$ pode ser administrada diariamente (Opinião).

6.9 Aumentar em 50\% a dose inicial de calcitriol se o PTH não diminuir em pelo menos $30 \%$ após 3 meses de tratamento, desde que os níveis de $\mathrm{Ca}$ e $\mathrm{P}$ estejam normais (Opinião). Os níveis séricos de $\mathrm{Ca}, \mathrm{P}$ e PTH devem ser medidos mensalmente por 3 meses.

6.10 O tratamento com vitamina $\mathrm{D}_{2}$ ou $\mathrm{D}_{3}$ ativa deve ser integrado com as alterações séricas de Ca, P e PTH (Opinião).

\section{Racional}

$\mathrm{Na}$ criança, o HPS ocorre quando a TFG é menor que $75 \mathrm{~mL} / \mathrm{min} / 1,73 \mathrm{~m}^{2}$. Nos estágios iniciais da DRC, se os níveis de PTH estão acima do limite da normalidade, a primeira medida é tratar a deficiência de 25-vit D; se os valores permanecerem elevados deve ser iniciado o tratamento com vitamina $\mathrm{D}_{2}$ ou $\mathrm{D}_{3}$ ativa (calcitriol). É recomendado manter níveis de PTH ligeiramente acima do normal em pacientes com DRC estágio IV, enquanto para pacientes em diálise as concentrações de PTH estar entre 3 a 5 vezes o limite superior da normalidade corresponde a taxas de formação óssea normais. ${ }^{1,4,5} \mathrm{~A}$ administração de doses baixas de calcitriol reduz os níveis séricos de PTH e melhora o crescimento linear, sem evidência de piora da função renal. É essencial monitorar os níveis séricos de Ca, P e PTH. Há evidência de que pacientes que recebem tratamento com vitamina $\mathrm{D}_{2}$ ou $\mathrm{D}_{3}$ ativa, quando o clearance de creatinina é maior que $30 \mathrm{~mL} / \mathrm{min} / 1,73 \mathrm{~m}^{2}$, atingem o estágio $\mathrm{V}$ da DRC apresentando histologia óssea normal. ${ }^{38} \mathrm{~A}$ deficiência de vitamina $\mathrm{D}$ ativa acarreta retardo de crescimento e doença óssea na criança com DRC; o tratamento com vitamina $\mathrm{D}_{2}$ ou $\mathrm{D}_{3}$ melhorou o crescimento linear em crianças com DRC estágios II a IV. ${ }^{39}$ Estes achados fornecem a racionalidade para a administração de 25-hidroxivitamina $\mathrm{D}$ ativa de rotina a praticamente todas as crianças com DRC. No entanto, doses altas

Tabela 11 ReComendações PARA A doSE INICIAL de CALCITRIOL EM CRIANÇAS COM DRC ESTÁGIO V

\begin{tabular}{|c|c|c|c|c|c|}
\hline PTH (pg/mL) & $\begin{array}{l}\text { Ca total } \\
(\mathrm{mg} / \mathrm{dL})\end{array}$ & $P(\mathrm{mg} / \mathrm{dL})$ & $\mathrm{Ca} \times \mathrm{P}$ & $\begin{array}{c}\text { Dose de calcitriol } \\
\text { oral - HD }\end{array}$ & $\begin{array}{c}\text { Dose de calcitriol } \\
\text { oral - DP }\end{array}$ \\
\hline $300-500$ & $<10$ & $\begin{array}{c}<5,5 \text { adolescentes } \\
<6,5 \text { lactentes } \\
\text { e crianças }\end{array}$ & $\begin{array}{c}<55 \text { adolescentes } \\
<65 \text { lactentes } \\
\text { e crianças }\end{array}$ & $\begin{array}{c}0,0075 \mu \mathrm{g} / \mathrm{kg} \\
\text { (máximo 0,25 } \mu \mathrm{g} \text { )/dia }\end{array}$ & $\begin{array}{c}0,0075 \mu \mathrm{g} / \mathrm{kg} \\
\text { (máximo 0,25 } \mu \mathrm{g} \text { )/dia }\end{array}$ \\
\hline$>$ 500-1.000 & $<10$ & $\begin{array}{c}<5,5 \text { adolescentes } \\
<6,5 \text { lactentes } \\
\text { e crianças }\end{array}$ & $\begin{array}{c}<55 \text { adolescentes } \\
<65 \text { lactentes } \\
\text { e crianças }\end{array}$ & $\begin{array}{c}0,015 \mu \mathrm{g} / \mathrm{kg} \\
\text { (máximo 0,5 } \mu \mathrm{g} \text { )/dia }\end{array}$ & $\begin{array}{c}0,015 \mu \mathrm{g} / \mathrm{kg} \\
\text { (máximo 0,5 } \mu \mathrm{g} \text { )/dia }\end{array}$ \\
\hline$>1.000$ & $<10,5$ & $\begin{array}{c}<5,5 \text { adolescentes } \\
<6,5 \text { lactentes } \\
\text { e crianças }\end{array}$ & $\begin{array}{c}<55 \text { adolescentes } \\
<65 \text { lactentes } \\
\text { e crianças }\end{array}$ & $\begin{array}{l}0,025 \mu \mathrm{g} / \mathrm{kg} / \mathrm{dia} \\
\text { (máximo } 1 \mu \mathrm{g} \text { ) }\end{array}$ & $\begin{array}{l}0,025 \mu \mathrm{g} / \mathrm{kg} / \mathrm{dia} \\
\text { (máximo } 1 \mu \mathrm{g} \text { ) }\end{array}$ \\
\hline
\end{tabular}

$\mathrm{HD}=$ hemodiálise; $\mathrm{DP}=$ diálise peritoneal. 
de 25-hidroxivitamina $\mathrm{D}$ ativa administradas de forma intermitente, associada a quelantes de P contendo $\mathrm{Ca}$, acomete a atividade da placa de crescimento epifisária e contribui para a redução do crescimento linear. ${ }^{40}$

A dose diária de calcitriol e alfacalcidol varia entre 0,25 a 1,5 $\mu \mathrm{g} /$ dia e é efetiva para controlar os níveis de PTH na maioria dos pacientes com DRC estágios II a IV. ${ }^{1}$

Pacientes com DRC em tratamento dialítico apresentam níveis séricos reduzidos de calcitriol, com consequente diminuição da absorção intestinal de $\mathrm{Ca}$ e aumento do PTH, acarretando o desenvolvimento do HPS. O tratamento com calcitriol melhora o HPS, a doença óssea e os sintomas musculoesqueléticos. ${ }^{41-43}$ Os efeitos colaterais mais frequentes são hipercalcemia e hiperfosfatemia, além da indesejada queda acentuada dos níveis séricos de PTH e desenvolvimento de doença óssea de baixa remodelação. Portanto, os níveis séricos de Ca, P e PTH devem ser monitorados durante a terapia com calcitriol e ajustados de acordo com as diretrizes. Pacientes em diálise com administração de 1,25-vit D via intravenosa ou pulso oral três vezes por semana mostrou-se efetiva para a redução dos níveis de PTH e permite o uso de doses mais altas de calcitriol sem o desenvolvimento de hipercalcemia quando administrado com quelantes de P sem Ca. ${ }^{1}$

O calcitriol (cápsula) pode não se mostrar efetivo quando o produto é aspirado da cápsula ou administrado através de sonda nasoenteral ou gastrostomias (pode ligar-se a materiais plásticos). Há formulações líquidas para esta administração que não estão disponíveis no mercado brasileiro.

Análogos da vitamina $\mathrm{D}_{2}$ ou $\mathrm{D}_{3}$ mais seletivos como alfacalcidol, paricalcitol ou doxercalciferol reduzem o risco de hipercalcemia e hiperfosfatemia. Estudo controlado, duplo-cego avaliou o efeito de paricalcitol $v s$. placebo por 12 semanas em um grupo de 29 pacientes em HD com idade entre 15 a 19 anos. A redução dos níveis de PTH foi superior no grupo paricalcitol $v s$. placebo, embora não tenham sido detectadas diferenças nos níveis séricos de $\mathrm{Ca}, \mathrm{P}$ ou produto $\mathrm{Ca} \times \mathrm{P} .{ }^{44}$ Estudo comparando o uso de paricalcitol vs. calcitriol em crianças em hemodiálise não detectou diferenças significativas entre os dois com relação à redução dos níveis de PTH e episódios de hipercalcemia. ${ }^{45}$ Portanto, não há dados convincentes que confirmem superioridade do efeito de uma 25-hidroxivitamina D específica sobre a outra.

Calcimiméticos: cinalcacet aumenta a sensibilidade ao Ca do receptor sensível ao Ca na glândula paratireoide e tem sido usado em adultos com DRC para suprimir a secreção do PTH com menor risco de hipercalcemia associado a calcitriol. As informações sobre seu uso e utilidade na criança são limitadas e não faz parte do tratamento padrão. Dois estudos observacionais com um total de 15 crianças em diálise e o outro com DRC grave relataram uma redução de $61 \%^{46}$ e de $74 \%{ }^{47}$ nos níveis séricos de PTH com cinalcacete.

\section{Concentração de Ca no dialisato}

7.1 A concentração padrão de Ca na solução de HD ou DP deve ser preferencialmente de 2,5 $\mathrm{mEq} / \mathrm{L}$ (Opinião).

7.2 Em pacientes em uso de quelantes de $\mathrm{P}$ contendo sais de $\mathrm{Ca}$, a concentração de $\mathrm{Ca}$ no dialisato deve ser mantida em 2,5 mEq/L. Naqueles que não estão em uso desses quelantes, a concentração de $\mathrm{Ca}$ no dialisato deve ser mantida em $3 \mathrm{mEq} / \mathrm{L}$, considerando-se os níveis séricos de $\mathrm{Ca}$ e a necessidade do tratamento com vitamina $\mathrm{D}_{2}$ ou $\mathrm{D}_{3}$ (Opinião).

\section{RACIONAL}

A infância e a adolescência são os períodos nos quais ocorre o maior incremento da massa óssea. Concentrações de Ca no dialisato de $2,5 \mathrm{mEq} / \mathrm{L}$ podem estar associadas com balanço negativo de $\mathrm{Ca}$, principalmente quando o paciente não está em uso de sais de Ca ou calcitriol. No entanto, a sobrecarga de $\mathrm{Ca}$ e consequente hipercalcemia favorece a CV e o desenvolvimento de doença adinâmica. ${ }^{20,48,49}$ Altas concentrações de $\mathrm{Ca}$ no dialisato aumentam a difusão de Ca para o paciente, enquanto níveis mais baixos favorecem o balanço negativo de $\mathrm{Ca}$. Concentrações de Ca no dialisato de 2,5 $\mathrm{mEq} / \mathrm{L}$ promovem balanço negativo de $\mathrm{Ca}$ em pacientes em $\mathrm{DP}^{50-52}$ assim como na HD. ${ }^{53-54}$ Pacientes em uso de quelantes de P contendo sais de $\mathrm{Ca}$ e/ou calcitriol podem desenvolver hipercalcemia. Portanto, o uso de concentração de Ca de 2,5 $\mathrm{mEq} / \mathrm{L}$ constitui uma estratégia adequada para evitar o balanço positivo de Ca. No entanto, o HPS pode agravar-se nessa condição, ${ }^{55} \mathrm{o}$ que pode ser minimizado por uma adequada ingestão de Ca. Pacientes 
que estão em uso de quelantes de $\mathrm{P}$ não contendo $\mathrm{Ca}$, e apresentam hipocalcemia mesmo após tratamento adequado com calcitriol, HPS refratário ou com síndrome da fome óssea pósparatireoidectomia devem ser dialisatos com concentração de $\mathrm{Ca}$ de 3,0 a 3,5 $\mathrm{mEq} / \mathrm{L}$. Não há estudos longitudinais que avaliem as diferentes concentrações de Ca no dialisato na população pediátrica. $\mathrm{O}$ uso de dialisato com concentração de Ca de 2,5 $\mathrm{mEq} / \mathrm{L}$ pode ser benéfico para prevenir hipercalcemia, doença óssea adinâmica e calcificação sistêmica, podendo, portanto, ser útil nas crianças que recebem quelantes de $\mathrm{P}$ contendo sais de Ca. É preciso considerar e monitorar o risco de hipocalcemia e HPS, assim como o crescimento linear.

\section{Acidose metabólica}

8.1 Os níveis séricos de $\mathrm{HCO}_{3}$ ou $\mathrm{CO}_{2}$ total devem ser monitorados na DRC.

8.1.1 A frequência das análises deve ser baseada nos estágios da DRC (Tabela 1) (Opinião).

8.2 Acidose metabólica deve ser corrigida seguindo as seguintes etapas:

8.2.1 Otimizar o tratamento dialítico - HD ou DP (Opinião).

8.2.2 Usar preferencialmente dialisato contendo bicarbonato para os pacientes em hemodiálise (Evidência).

8.2.3 Na DP as soluções contendo bicarbonato com pH neutro (bolsas com dois compartimentos) são mais biocompatíveis e melhoram a acidose metabólica (Opinião).

8.2.3 Alguns equipamentos de HD possibilitam modular o bicarbonato durante a diálise.

8.2.4 Administrar bicarbonato de sódio por via oral na dose de 2 a $3 \mathrm{mEq} / \mathrm{kg} / \mathrm{dia}$, caso as medidas anteriores não corrijam a acidose metabólica (Opinião).

\section{RACIONAL}

A acidose metabólica é comum desde os estágios iniciais da DRC. Ela é causada pela deficiência de excreção renal de íons hidrogênio e consequente acúmulo de produtos ácidos do metabolismo. Caracteriza-se por redução do $\mathrm{pH}$ e dos níveis séricos de bicarbonato. Valores de bicarbonato sérico acima de $20 \mathrm{mEq} / \mathrm{L}$ são normais para recém-nascidos e lactentes abaixo de 2 anos de idade; para crianças acima de 2 anos o limite inferior de normalidade é $22 \mathrm{mEq} / \mathrm{L} .{ }^{4} \mathrm{~A}$ acidose pode acarretar hipercalemia, uma vez que para uma queda do $\mathrm{pH}$ de 0,1 há aumento dos níveis séricos de potássio de $0,7 \mathrm{mmol} / \mathrm{L}$, pois os íons de hidrogênio são captados pela célula em troca por potássio.

A acidose tem efeito adverso sobre o esqueleto. $\mathrm{O}$ tamponamento ósseo dos íons hidrogênio aumenta a liberação de Ca e P. A acidose metabólica crônica aumenta a reabsorção óssea e inibe a formação endocondral $^{56}$ e é causa de retardo de crescimento na criança, mesmo com função renal preservada. ${ }^{57}$ $\mathrm{O}$ aumento da reabsorção óssea libera carbonato e fosfato do mineral ósseo e a redução da formação óssea diminui a quantidade de ácido produzida durante a mineralização óssea. ${ }^{58} \mathrm{~A}$ correção da acidose metabólica permite a normalização do crescimento linear em crianças com acidose tubular isolada. Os efeitos da acidose metabólica sobre o crescimento ocorrem por meio das alterações na mineralização óssea, no eixo IGF-1/GH (hormônio de crescimento) e na síntese renal de calcitriol. ${ }^{57} \mathrm{~A}$ acidose crônica contribui na gênese do HPS porque reduz a síntese de 1,25-vit $\mathrm{D}$ pelo túbulo proximal e pode limitar a absorção dietética de $\mathrm{Ca} .{ }^{4} \mathrm{~A}$ acidose também estimula a proliferação celular da paratireoide. Acidose e PTH, de forma independente, estimulam a saída de Ca do osso, inibem a síntese osteoblástica de colágeno e estimulam a secreção osteoclástica de $\beta$-glucoronidase. Portanto, na presença de acidose + PTH, há uma ação maior em cada um destes parâmetros em comparação ao efeito isolado, sugerindo um efeito deletério aditivo do PTH e da acidose sobre o osso. ${ }^{58}$ Dessa forma, na criança com DRC a acidose metabólica contribui para o desenvolvimento da doença óssea, sendo o raquitismo a lesão mais frequente na criança com DRC estágios I a III. ${ }^{4} \mathrm{~A}$ recomendação do grupo de estudo do KDOQI é de manter os níveis séricos de bicarbonato > $22 \mathrm{mEq} / \mathrm{L}$; a terapia com bicarbonato de sódio deve ser iniciada na dose de 1 a $2 \mathrm{mEq} / \mathrm{kg} /$ dia dividido em 2 a 3 doses, e a dose ajustada para atingir os valores-alvo. O uso de citrato deve ser cauteloso na criança com DRC pelo risco de intoxicação alumínica. ${ }^{2}$

A acidose aumenta o catabolismo proteico, piorando os sintomas de uremia. O aumento do catabolismo muscular aumenta a necessidade da cota proteica dietética para manter o balanço de nitrogênio neutro. Dessa forma, a acidose piora o estado nutricional já comprometido na criança com DRC. 


\section{Tratamento da doença óssea na DRC}

Hiperparatireoidismo secundário (Doença óssea de alta remodelação)

9.1. Para pacientes nos estágios II e III da DRC e PTH maior que $70 \mathrm{pg} / \mathrm{mL}$ ou no estágio IV e PTH maior que $110 \mathrm{pg} / \mathrm{mL}$, o aporte de $\mathrm{P}$ deve ser modificado de acordo com as Diretrizes 3 e 4 e a ingestão de Ca de acordo com a Diretriz 2 (Opinião).

9.2 A deficiência de 25-vit D deve ser corrigida de acordo com a Diretriz 5. Se os níveis séricos de PTH permanecerem elevados após 3 meses da intervenção dietética, iniciar a administração de calcitriol (Opinião).

9.3 Na DRC estágio V e PTH maior que 300 $\mathrm{pg} / \mathrm{mL}$, apesar das medidas recomendadas nas Diretrizes 2, 3, 4 e 5, a administração de calcitriol deve ser iniciada (Evidência).

\section{RACIONAL}

Enquanto nos adultos com DRC a doença óssea se manifesta, geralmente, no estágio III, na criança a manifestação pode ocorrer mais precocemente, ainda no estágio II, pela maior incidência de tubulopatias com acidose, distúrbios causadores de perda de P e má nutrição. ${ }^{59}$ Crianças com doença óssea apresentam retardo de crescimento, deformidades das extremidades, deslizamento epifisário e fraturas; esses sintomas podem estar presentes precocemente ainda com função renal relativamente preservada. Na criança, devido aos riscos de doença óssea persistente e autonomia da glândula paratireoide, deve-se evitar a elevação dos níveis de PTH acima dos valores recomendados. Portanto, a elevação dos níveis séricos de PTH deve ser seguida pela instituição das medidas de restrição de $\mathrm{P}$ (dieta e uso de quelantes). Essas medidas se associam à normalização dos níveis séricos de FA, PTH e manutenção da remodelação óssea normal, ${ }^{60}$ assim como à melhora do crescimento em lactentes e pré-escolares. ${ }^{61}$

\section{Raquitismo/Osteomalacia}

9.4 Osteomalacia por intoxicação por alumínio (Al) deve ser prevenida em pacientes com DRC evitando-se concentrações de Al no dialisato acima de $10 \mu \mathrm{g} / \mathrm{L}$, assim como o uso oral de compostos de Al (Evidência).
9.5 Raquitismo e osteomalacia devido à deficiência de 25-vit D devem ser tratados de acordo com a Diretriz 5 (Opinião).

9.6 Raquitismo e osteomalacia causados por hipofosfatemia devem ser tratados com sais de fosfato neutro. Considerar também a terapia com calcitriol. Consultar Diretrizes 2 e 5 (Evidência).

\section{RACIONAL}

A intoxicação alumínica, apesar de estar associada às doenças ósseas de baixa remodelação, pode cursar com todos os tipos de doença óssea na DRC. $\mathrm{Na}$ criança, a intoxicação por alumínio causa sintomas como dor óssea, deformidades ósseas, redução do crescimento e até mesmo manifestações neurológicas, como convulsão. Embora a exposição do paciente ao Al tenha reduzido substancialmente nas duas últimas décadas, a doença óssea associada ao Al ainda pode ocorrer e este diagnóstico deve ser considerado. Raquitismo e osteomalacia também podem estar presentes em crianças na ausência de intoxicação por Al. Raquitismo corresponde a uma falha ou retardo da mineralização do osso endocondral recém-formado situado nas placas de crescimento, enquanto osteomalacia é caracterizada pela falha da mineralização do osteoide recém-formado nos locais de remodelamento ósseo ou aposição periosteal ou endosteal. A osteomalacia que ocorre na ausência de intoxicação por Al se deve à hipofosfatemia, à deficiência de vitamina $\mathrm{D}_{2}$ ou $\mathrm{D}_{3}$, acidose metabólica e deficiência de Ca e P. ${ }^{1}$

\section{Doença óssea adinâmica}

9.7 Na DRC estágio $\mathrm{V}$, a doença óssea adinâmica não relacionada ao $\mathrm{Al}$ (determinada pela biópsia óssea ou pelos níveis séricos de PTH $<150 \mathrm{pg} / \mathrm{mL}$ ) deve ser tratada com medidas que propiciem uma elevação dos níveis de PTH para restabelecer a remodelação óssea (Opinião).

9.8 A otimização dos níveis de PTH pode ser atingida pela suspensão do uso de calcitriol, redução ou suspensão dos quelantes de $\mathrm{P}$ contendo $\mathrm{Ca}$, redução da concentração de Ca do dialisato (Diretrizes 7) (Evidência) e/ou quelante de $\mathrm{P}$ não contendo $\mathrm{Ca}$ (Opinião). 


\section{Racional}

$\mathrm{O}$ uso frequente de calcitriol e de quelantes de $\mathrm{P}$ contendo Ca tem contribuído para o aumento da prevalência da doença óssea adinâmica ${ }^{62}$ que comumente está associada a níveis séricos de PTH abaixo de 150 $\mathrm{pg} / \mathrm{mL}$. As consequências clínicas da doença óssea adinâmica são o maior risco de fraturas ósseas, redução do crescimento linear e a incapacidade do osso adinâmico em manter a homeostase mineral. A manifestação clínica da doença óssea adinâmica na criança com DRC estágio V não está bem caracterizada. No entanto, sabe-se que a sobrecarga de Ca proveniente dos quelantes de $\mathrm{P}$ favorece calcificação extraóssea em tecidos moles e vasos. ${ }^{63,64}$

\section{Avaliação do crescimento e recomendações para o uso do hormônio de crescimento (rhGH)}

\section{DRC III a V}

10.1 Todas as crianças devem ter monitorada a taxa de crescimento com medida da altura em centímetros e determinado o escore $\mathrm{Z}$ para altura, com uma frequência trimestral para os lactentes nos estágios de DRC II a V e, pelo menos, anual nas criança (Opinião).

10.2 Crianças e adolescentes com DRC estágios II a $\mathrm{V}$ e com déficit de altura relacionado à DRC é recomendado o tratamento com hormônio de crescimento recombinante $(\mathrm{rhGH})$ quando se deseja crescimento adicional, após a correção da má nutrição e das alterações bioquímicas da DMO-DRC (Opinião).

10.3 Antes do início do tratamento com rhGH deve-se corrigir o aporte proteico-energético, a acidose metabólica, a hiperfosfatemia e o HPS (Evidência).

\section{RACIONAL}

O retardo do crescimento ocorre com frequência de $11,5 \%$ a $13 \%$ na criança com DRC em todos os estágios, tendo prevalência crescente nos estágios mais avançados. ${ }^{62-67}$ Sua etiologia é multifatorial e, além da osteodistrofia renal, inclui ingestão alimentar insuficiente, alteração no paladar pela deficiência de zinco, uremia, dieta pobre em sódio, potássio e $\mathrm{P}$, anemia, acidose metabólica, deficiência de 25 -vit $\mathrm{D}$ e fatores relacionados à diálise. Anormalidades endócrinas, como resistência tecidual ao IGF-1, resistência à insulina, hiperglucagonemia, HPS, alterações no eixo
GH/IGF, associado à insensibilidade ao GH e deficiência funcional do IGF-1 também estão implicados. ${ }^{68}$ Medidas terapêuticas como uso de vitamina $\mathrm{D}_{2}$ ou $\mathrm{D}_{3}$ e normalização da concentração de PTH melhoram o crescimento (Walker e cols., 2003). Uma revisão sobre o uso do rhGH em crianças com DRC avaliou 15 estudos com 629 crianças e comparou rhGH com placebo. ${ }^{69}$ Esses estudos mostraram melhora nos parâmetros de crescimento com resultados positivos em todos os aspectos e por um tempo de avaliação de 6 até 24 meses. Os dados disponíveis sugerem que a criança deve ser tratada com uma dose de 28 IU/ $\mathrm{m}^{2} /$ semana. A consistência dos benefícios do uso de rhGH mostrada nos diversos estudos considera este uso como evidência de alta qualidade, levando a uma forte recomendação para seu uso na criança com DRC e déficit de crescimento. ${ }^{2}$ Antes de iniciar o tratamento com $\mathrm{GH}$, os níveis séricos de $\mathrm{P}$ devem estar abaixo de 1,5 vez o limite superior para a idade e PTH abaixo de 1,5 vez o limite superior para o estágio da DRC. ${ }^{4} \mathrm{O}$ tratamento com $\mathrm{GH}$ eleva os níveis de PTH durante os primeiros meses de tratamento e, portanto, os níveis de PTH devem ser monitorados mensalmente e o uso de GH suspenso caso os níveis de PTH excedam três vezes o limite superior para o estágio da DRC. ${ }^{4} \mathrm{O}$ uso de rhGH melhora o crescimento linear de crianças com DRC pré-diálise, HD e DP, ${ }^{70,71}$ aumenta os níveis de IGF-1 e melhora a densidade mineral óssea. ${ }^{72,73}$ A resposta é melhor nas crianças em tratamento conservador, cuja função renal está mais preservada. ${ }^{74}$ Estudos randomizados com um número maior de crianças, como o Southwest Pediatric Nephrology Study Group, compovaram a eficácia do tratamento com rhGH..$^{75,76}$

\section{Indicação de biópsia óssea}

11.1 O diagnóstico da doença óssea deve ser feito pela biópsia óssea obtida na crista ilíaca seguida de análise histomorfométrica (Evidência).

11.2 A biópsia óssea pode estar indicada no estágio $\mathrm{V}$, não sendo geralmente necessária nos estágios I a IV, exceto quando houver suspeita de osteomalácia (Evidência).

\section{RAcional}

A biópsia óssea fornece informações sobre a remodelação e mineralização ósseas, assim como sobre a presença de metais, como ferro e alumínio. A análise 
histomorfométrica completa exige a marcação prévia pela tetraciclina, um marcador da mineralização óssea. A tetraciclina deve ser administrada em duas doses de $15 \mathrm{mg} / \mathrm{kg} / \mathrm{dia}$, em dois períodos de 3 dias, separados por um intervalo de 10 dias. Em crianças menores de 8 anos, a dose deve ser menor que $10 \mathrm{mg} / \mathrm{kg} / \mathrm{dia}$. A biópsia óssea deve ser considerada na presença de fraturas sem ou com mínimo trauma (fraturas patológicas) (Opinião), suspeita de doença óssea por alumínio baseado em sintomas clínicos ou evidência de exposição ao metal (Opinião), hipercalcemia persistente com níveis séricos de PTH entre 400-600 pg/mL. ${ }^{1,2,4,26}$

\section{REFERÊNCIAS}

1. Bakkaloglu SA, Wesseling-Perry K, Salusky IB. Chronic Kidney disease-mineral and bone disorder (CKD_MBD) in children. In: Olgard K, Salusky IB, Silver J (eds.). The Spectrum of Mineral and Bone Disorders in Chronic Kidney Disease. 2.ed. New York: Oxford University Press, 2010; p 485-507.

2. KDIGO Clinical Practice Guideline for the Diagnosis, Evaluation, Prevention, and Treatment of Chronic Kidney Disease-Mineral and Bone Disorder (CKDMBD). Kidney Int. 2009; 76, S1-S130.

3. Gutierrez $\mathrm{O}$, Isakova $\mathrm{T}$, Rhee $\mathrm{E}$ et al. Fibroblast growth factor-23 mitigates hyperphosphatemia but accentuates calcitriol deficiency in chronic kidney disease. J Am Soc Neprol. 16(7):2205-15.

4. K/DOQI Clinical Practice Guidelines for Bone Metabolism and Disease in Children With Chronic Kidney Disease. Am J Kidney Disease 2005; 46(Suppl 1):S1-S121.

5. Wesseling K, Bakkaloglu S, Salusky I. Chronic Kidney disease mineral and bone disorder in children. Pediatr Nephrol. 2008; 23:195-207.

6. Block GA. Prevalence and clinical consequences of elevated $\mathrm{Ca} \times \mathrm{P}$ product in hemodialysis patients. Clin Nephrol. 2000; 54(4):318-24.

7. Salusky IB, Ramirez JA, Oppenheim W, Gales B, Segre GV, Goodman WG. Biochemical markers of renal osteodystrophy in pediatric patients undergoing CAPD/ CCPD. Kidney Int. 1994; 45:253-8.

8. Mathias R, Salusky I, HarmanW et al. Renal bone disease in pediatric and young adult patients on hemodialysis in a children's hospital. J Am Soc Nephrol. 1993; 3:938-46.

9. Portale AA. Blood calcium, phosphorus, and magnesium, In Primer on the Metabolic Bone Diseases and Disorders of Mineral Metabolism, Favus MJ (ed.). Philadelphia: Lippincott, Williams \&Wilkins, 1999; pp. 115-18.

10. RDI Institute of Medicine. Dietary references intakes: calcium, phosphorus, magnesium, vitamin D3, and fluoride. Washington, D.C.: National Academy Press, 2000.

11. Hercz G, Kraut JA, Andress DA et al.Use of calcium carbonate as a phosphate binder in dialysis patients. Miner Electrolyte Metab. 1986; 12:314-19.
12. Slatoplsky E, Weerts C, Lopes-Hilker S et al. Calcium carbonate as a phosphate binder in patients with chronic renal failure undergoing dialysis. $\mathrm{N}$ Engl J Med. 1986; 315:157-61.

13. Moriniere P, Fournier A, Leflon A et al. Comparison of $1 \alpha$ alfa-OH-vitamin D3 and high doses of calcium carbonate for the control of hyperparathyroidism and hyperaluminemia in patients with chronic renal failure undergoing dialysis. Nephron. 1985; 39:309-15.

14. Shane E. Hypercalcemia; pathogenesis, clinical manifestation, differential diagnosis and management, in Primer on the Metabolic Bone Diseases and Disorders of Mineral Metabolism, Favus MJ (ed.). Philadelphia: Lippincott, Williams \&Wilkins, 1999; pp. 183-87.

15. Massry SG, Smogorzewski M. Dyscalcemias. In: Massry SG, Glassock RJ (eds.). Massry and Glassock's Textbook of Nephrology. Philadelphia: Lippincott, Williams \& Wilkins, 2001; pp. 326-40.

16. Raper NR, Zissa C, Rourke J. Nutrient Content of the US Food Supply, 1909-1988, Washington, D.C.: US Dept of Agriculture, 1992 (Home Economics Research Report No. 50).

17. National Kidney Foundation. K/DOQI clinical practice guidelines for nutrition in chronic renal failure: 2008 Update. Am J Kidney Dis. 2009; 53(Suppl2):S1.

18. Brodehl J, Gellissen K, Weber HP. Postnatal development of tubular phosphate reabsorption. Clin Nephrol. 1982; 17:163-71.

19. Greenberg BG, Winters RW, Graham JB. The normal range of serum inorganic phosphorus and its utility as a discriminant in the diagnosis of congenital hypophosphatemia. J Clin Endocrinol Metab. 1960; 20:364-79.

20. Goodman WG, Goldin J, Kuizon BD et al.Coronary artery calcification in young adults with end-stage renal disease who are undergoing dialysis. N Engl J Med. 2000; 342:1478-83.

21. Block GA, Klassen PS, Lazarus JM, Ofsthun N, Lowrie EG, Chertow GM. Mineral metabolism, mortality, and morbidity in maintenance hemodialysis. J Am Soc Nephrol. 2004; 15:2208-18.

22. Moe S, Drüeke T, Cunningham J et al. Definition, evaluation, and classification of renal osteodystrophy: a position statement from Kidney Disease: Improving Global Outcomes (KDIGO). Kidney Int. 2006; 69:1945-53.

23. Portale AA, Booth BE, Halloran BP, Morris RC Jr. Effect of dietary phosphorus on circulating concentrations of 1,25-dihydroxyvitamin D and immunoreactive parathyroid hormone in children with moderate renal insufficiency. J Clin Invest. 1984; 73:1580-9.

24. Voormolen N, Noordzij M, Grootendorst DC et al. The PREPARE study group. High plasma phosphate as a risk factor for decline in renal function and mortality in pre-dialysis patients. Nephrol Dial Transplant. 2007; 22(10): 2909-16.

25. Schwarz S, Trivedi BK, Kalantar-Zadeh K, Kovesdy CP. Association of disorders in mineral metabolism with progression of chronic kidney disease. Clin J Am Soc Nephrol. 2006; 1:825-31.

26. Klaus G, Watson A, Edefonti A et al. European Pediatric Dialysis Working Group (EPDWG). Prevention and treatment of renal osteodystrophy in children on chronic renal failure: European guidelines. Pediatr Nephrol. 2006; 21(2):151-9. 
27. Salusky IB. A new era in phosphate binder therapy: What are the options? Kidney Int. 2006; 105(Suppl.):S10-15.

28. Storms LE, Chicella MF, Dice JE. Sevelamer therapy for pediatric end-stage renal disease. Pharmacotherapy 2006; 26(3):410-13.

29. Pieper AK, Haffner D, Hoppe B et al. A randomized crossover trial comparing sevelamer with calcium acetate in children with CKD. Am J Kidney Dis. 2006; 47:625.

30. Mahdavi H, Kuizon BD, Gales B, Wang HJ, Elashoff RM, Salusky IB. Sevelamer hydrochloride: an effective phosphate binder in dialyzed children. Pediatr Nephrol. 2003; 18:1260-64.

31. Gonzalez E, Schomberg J, Amin N et al. Sevelamer carbonate increases serum bicarbonate in pediatric dialysis patients. Pediatr Nephrol. 2010; 25:373.

32. Ali FN, Arguelles LM, Langman CB, Price HE, Vitamin $\mathrm{D}$ deficiency in children with chronic kidney disease: uncovering an epidemic. Pediatrics 2009; 123:791-6.

33. Linhares ER, Jones DA, Round JM, Edwards RHT. Effect of nutrition on vitamin D status: studies on healthy and poorly nourished Brazilian children. Am J Clin Nutr. 1984; 39:630-45.

34. Maeda SS, Kunii IS, Hayashi L, Lazaretti-Castro M. The effect of sun exposure on 25-hydroxyvitamin D concentrations in young healthy subjects living in the city of São Paulo, Brazil. Braz J Med Biol Res. 2007; 40(12):1653-9.

35. Guillemant J, Cabrol S, Allemandou A, Peres G, Guillemant S. Vitamin D-dependent seasonal variation of PTH in growing male adolescents. Bone 1995; 17:513-16.

36. Seeherunvong W, Abitbol CL, Chandar J et al. Vitamin $\mathrm{D}$ insufficiency and deficiency in children with early chronic kidney disease. J Pediatr. 2009; 154:906.

37. Holick MF. Vitamin D deficiency. N Engl J Med. 2007; 357:266-81.

38. Nordal KP, Dahl E, Halse J, Attramadal A, Flatmark A. Long-term low-dose calcitriol treatment in predialysis chronic renal failure: can it prevent hyperparathyroid bone disease? Nephrol Dial Transplant, 1995; 10:203-6.

39. Chesney RW, Moorthy AV, Eisman JA, Jax DK, Mazess $\mathrm{RB}$, DeLuca HF. Increased growth after long-term oral 1alpha,25-vitamin D3 in childhood renal osteodystrophy. N Engl J Med. 1978; 298:238-42.

40. Kuizon BD, Goodman WG, Juppner Het al. Diminished linear growth during intermittent calcitriol therapy in children undergoing CCPD. Kidney Int. 1998; 53:205-11.

41. Brickman AS, Coburn JW, Sherrard DJ, Wong EG, NormanAW, Singer FR. Clinical effects of 1,25-dihydroxyvitamin D3 in uremic patients with overt osteodystrophy. Contrib Nephrol. 1980; 18:29-41.

42. Moriniere P, Esper NE, Viron B et al. Improvement of severe secondary hyperparathyroidism in dialysis patients by intravenous 1 -alpha $(\mathrm{OH})$ vitamin $\mathrm{D} 3$, oral $\mathrm{CaCO} 3$ and low dialysate calcium. Kidney Int. 1993; 43(supp141):S121-S4.

43. Sherrard DJ, Coburn JW, Brickman AS, Singer FR, Maloney N. Skeletal response to treatment with 1,25-dihydroxyvitamin $\mathrm{D}$ in renal failure. Contrib Nephrol. 1980; 18:92-7.
44. Greenbaum LA, Benador N, Goldstein SL et al. Intravenous paricalcitol for treatment of secondary hyperparathyroidism in children on hemodialysis. Am J Kidney Dis. 2007; 49:814.

45. Seeherunvong W, Nwobi O, Abitbol CL et al. Paricalcitol versus calcitriol treatment for hyperparathyroidism in pediatric hemodialysis patients. Pediatr Nephrol. 2006; 21:1434.

46. Muscheites J, Wigger M, Drueckler E, Fischer DC, Kundt G, Haffner D. Cinacalcet for secondary hyperparathyroidism in children with end-stage renal disease. Pediatr Nephrol. 2008; 23(10):1823-9.

47. Silverstein DM, Kher KK, Moudgil A, Khurana M, Wilcox J, Moylan K. Cinacalcet is efficacious in pediatric dialysis patients. Pediatr Nephrol. 2008; 23(10):1817-22.

48. Guerin AP, London GM, Marchais SJ, Metivier F. Arterial stiffening and vascular calcifications in end-stage renal disease. Nephrol Dial Transplant. 2000; 15:1014-21.

49. Goodman WG, Ramirez JA, Belin TR et al. Development of adynamic bone in patients with secondary hyperparathyroidism after intermittent calcitriol therapy. Kidney Int. 1994; 46:1160-6.

50. Piraino B, Bernardini J, Holley J, Johnston JR, Perlmutter JA, Martis L. Calcium mass transfer in peritoneal dialysis patients using $2.5 \mathrm{mEq} / \mathrm{l}$ calcium dialysate. Clin Nephrol. 1992; 37:48-51.

51. Malberti F, Corradi B, Imbasciati E. Calcium mass transfer and kinetics in CAPD using calcium-free solutions. Adv Perit Dial. 1993; 9:274-9.

52. Sieniawska M, Roszkowska-Blaim M, Wojciechowska B. The influence of dialysate calcium concentration on the PTH level in children undergoing CAPD. Perit Dial Int. 1996; 16(suppl1):S567-9.

53. Fabrizi F, Bacchini G, Di Filippo S, Pontoriero G, Locatelli F. Intradialytic calcium balances with different calcium dialysate levels. Effects on cardiovascular stability and parathyroid function. Nephron. 1996; 72:530-35.

54. Fernandez E, Borras M, Pais B, Montoliu J. Low calcium dialysate stimulates parathormone secretion and its long-term use worsens secondary hyperparathyroidism. J Am Soc Nephrol. 1995; 6:132-5.

55. Buijsen CG, Struijk DG, Huijgen HJ, Boeschoten EW, Wilmink JM. Can low-calcium peritoneal dialysis solution safely replace the standard calcium solution in the majority of chronic peritoneal dialysis patients? Perit Dial Int. 1996; 16:497-504.

56. Lefebvre A, de Vernejoul MC, Gueris J, Goldfarb B, Graulet AM, Morieux C. Optimal correction of acidosis changes progression of dialysis osteodystrophy. Kidney Int. 1989; 36(6):1112-8.

57. McSherry E, Morris RC Jr. Attainment and maintenance of normal stature with alkali therapy in infants and children with classic renal tubular acidosis. J Clin Invest. 1978; 61(2):509-27.

58. Bushinsky DA. Acidosis and renal bone disease. In: Olgard K, Salusky IB, Silver J (eds.). The Spectrum of Mineral and Bone Disorders in Chronic Kidney Disease. 2.ed. New York: Oxford University Press, 2010; p.253-65.

59. Langman CB. Renal osteodystrophy: a pediatric perspective, 2005. Growth Horm IGF Res. 2005; 15(Suppl A):42-7. 
60. Walter S, Reynolds A, Ridout D, Cantor T, Gao P, Rees L. Parathyroid hormone and its fragments in children with chronic renal failure. Pediatr Nephrol. 2003; 18:1242-8.

61. Walter S, Ledermann S, Trompeter R, Van't Hoff W, Ridout D, Rees L. Catch-up growth with normal parathyroid hormone levels in chronic renal failure. Pediatr Nephrol. 2003; 18:1236-41.

62. Salusky IB, Goodman WG. Adynamic renal osteodystrophy: is there a problem? J Am Soc Nephrol. 2001; 12:1978-85.

63. London GM, Marty C, Marchais SJ, Guerin AP,Metivier F, de Vernejoul MC. Arterial calcifications and bone histomorphometry in end-stage renal disease. J Am Soc Nephrol. 2004; 15:1943-51.

64. Querfeld U. The clinical significance of vascular calcification in young patients with end-stage renal disease. Pediatr Nephrol. 2004; 19: 478-84.

65. Wong H, Mylrea K, Feber J, Drukker A, Filler G. Prevalence of complications in children with chronic kidney disease according to KDOQI. Kidney Int. 2006; 70:585-90.

66. Fivush BA, Jabs K, Neu AM et al. Chronic renal insufficiency in children and adolescents: the 1996 annual report of NAPRTCS. North American Pediatric Renal Transplant Cooperative Study. Pediatr Nephrol. 1998; 12:328-37.

67. Rees L, Rigden SP, Ward GM. Chronic renal failure and growth. Arch Dis Child. 1989; 64:573-7.

68. Baumann G. Growth hormone binding protein and free growth hormone in chronic renal failure Pediatric Nephrol. 1996; 10(3):328-30.

69. Vimalachandra D, Hodson EM, Willis NS, Craig JC, Cowell C, Knight JF. Growth hormone for children with chronic kidney disease. Cochrane Database Syst Rev. 2006 Jul 9; 3:CD003264.
70. Fine RN, Kohaut E, Brown D, Kuntze J, Attie KM. Long-term treatment of growth retarded children with chronic renal insufficiency, with recombinant human growth hormone Kidney Int. 1996; 49(3):781-5.

71. Koch VH, Lippe BM, Nelson PA, Boechat MI, Shermen BM, Fine RN. Accelerated growth after recombinant human growth hormone treatment of children with chronic renal failure. J Pediatr 1989; 115(3):365-71.

72. Hokken-Koelega A, Mulder P, De Jong R, Lilien M, Donckerwolcke R, Groothof J. Long-term effects of growth hormone treatment on growth and puberty in patients with chronic renal insufficiency Dutch randomized GH trials of children with growth retardation secondary to CRI. Pediatr Nephrol. 2000; 14:701-6.

73. Van Dyck M, Gyssels A, Proesmans W, Nijs J, Eeckels R. Growth hormone treatment enhances bone mineralization in children with chronic renal failure. Eur J Pediatr. 2001; 160:359-63.

74. Wühl E, Haffner D, Nissel F. Short dialysed children respond less to growth hormone than patients prior to dialysis. German Study Group for Growth Hormone Treatment in Chronic Renal Failure. Pediatr Nephrol. 1996; 10(3):294-8.

75. Powell DR, Liu F, Baker BK et al. Modulation of growth factors by growth hormone in children with chronic renal failure. The Southwest Pediatric Nephrology Study Group. Kidney Int. 1997; 51:1970-9.

76. Bérard E, Crosnier H, Six-Beneton A, Chevallier T, Cochat T, Broyer M. Recombinant human growth hormone treatment of children on hemodialysis. French Society of Pediatric Nephrology. Pediatr Nephrol. 1998; 12:304-10. 